\title{
Classificação de famílias do feijoeiro sob diferentes cenários de dependência espacial e precisão experimental
}

\author{
Josmar Furtado de Campos $^{(1)}$, Antonio Policarpo Souza Carneiro(1), Luiz Alexandre Peternelli(1), \\ José Eustáquio de Souza Carneiro(2), Michele Jorge da Silva(2) e Paulo Roberto Cecon ${ }^{(1)}$
}

(1)Universidade Federal de Viçosa (UFV), Departamento de Estatística, Avenida Peter Henry Rolfs, s/no, CEP $36570-000$ Viçosa, MG, Brasil.
E-mail: josmarfc@yahoo.com.br, policarpo@ufv.br, peternelli@ufv.br, cecon@ufv.br (2)UFV, Departamento de Fitotecnia, Avenida Peter Henry
Rolfs, s/n־, CEP 36570-000 Viçosa, MG, Brasil. E-mail: jesc@ufv.br, michelejorgesilva@gmail.com

Resumo - O objetivo deste trabalho foi avaliar a eficiência da análise estatística espacial, em comparação a análises usuais em blocos ao acaso e em látice, na classificação de famílias de feijoeiro (Phaseolus vulgaris), sob diferentes cenários de dependência espacial e de precisão experimental. Foram considerados 12 cenários, formados por quatro classes de dependência espacial entre erros (nula, baixa, média e alta) e três classes de precisão experimental (moderada, alta e muito alta). Para as três classes de precisão experimental, foram definidos os valores de acurácia seletiva de: $0,60,0,80$ e 0,95 , respectivamente. Já as quatro classes de dependência espacial entre erros assumiram, respectivamente, os seguintes valores de alcance do modelo geoestatístico exponencial: $0,10,20$ e $40 \mathrm{~m}$. Para experimentos com precisão experimental muito alta ou ausência de dependência espacial, a eficiência da análise espacial para classificação de famílias do feijoeiro é semelhante à eficiência das análises usuais. Para experimentos com alta e, principalmente, com moderada precisão experimental, a análise espacial é mais eficiente que as análises usuais para classificação de famílias de feijoeiro, quando os erros apresentam dependência espacial.

Termos para indexação: Phaseolus vulgaris, acurácia seletiva, análise espacial, autocorrelação, erros dependentes, modelos geoestatísticos.

\section{Classification of common bean families under different scenarios of spatial dependence and experimental precision}

\begin{abstract}
The objective of this work was to evaluate the efficiency of spatial statistical analysis, compared to the usual analyzes in randomized blocks and in lattice, for the classification of common bean (Phaseolus vulgaris) families under different scenarios of spatial dependence and experimental precision. Twelve scenarios were considered, formed by four classes of spatial dependence between errors (null, low, medium, and high) and three classes of experimental precision (medium, high, and very high). For the three classes of experimental precision, the following values of selective accuracy were defined: $0.60,0.80$, and 0.95 , respectively. The four classes of spatial dependence between errors assumed, respectively, the following range values for the exponential geostatistical model: $0,10,20$, and $40 \mathrm{~m}$. In experiments with very high experimental precision or with the absence of spatial dependence, the efficiency of the spatial analysis for the classification of common bean families is similar to that of the usual analyses. In experiments with high and, especially, with medium experimental precision, the spatial analysis is more efficient than the usual analyses for the classification of common bean families, when the errors show spatial dependence.
\end{abstract}

Index terms: Phaseolus vulgaris, selective accuracy, spatial analysis, autocorrelation, dependent errors, geostatistical models.

\section{Introdução}

Nos programas de melhoramento do feijoeiro (Phaseolus vulgaris L.), a fase inicial de seleção de famílias envolve grande número de genótipos, o que exige extensas áreas experimentais. $\mathrm{Na}$ maioria das vezes, o delineamento em blocos ao acaso torna-se inviável em razão da alta heterogeneidade dentro dos blocos, uma vez que eles devem comportar grande número de famílias. Assim, o delineamento em látice é comumente utilizado para aumentar a precisão experimental dos ensaios de avaliação genética do feijoeiro (Ramalho et al., 2012). No entanto, 
a instalação de ensaios em látice apresenta pouca flexibilidade quanto ao número de famílias avaliadas, que deve apresentar raiz quadrada exata. Além disso, nas análises em bloco ou em látice, assume-se que os erros são espacialmente independentes, o que nem sempre ocorre (Costa et al., 2005; Duarte \& Vencovsky, 2005; Candido et al., 2009; Feres, 2009).

Os métodos de análise estatística que levam em conta a posição das parcelas na área experimental são denominados de métodos de análise espacial. A aplicação desses métodos busca aumentar a eficiência do controle local e a precisão experimental. Nesse tipo de análise, considera-se que os erros das parcelas experimentais são tão mais semelhantes, quanto mais próximas elas estejam entre si. Desse modo, considerase a existência de uma relação de dependência espacial entre os erros (Costa et al., 2005).

Segundo Cressie \& Hartfield (1996), a dependência espacial tem sido estudada desde meados de 1930. Os autores mencionam que estimadores precisos dos efeitos de tratamentos podem ser obtidos, em ensaios de campo, de duas maneiras: a primeira, e mais comum, é utilizar delineamentos experimentais para controle local, como blocos ao acaso, linha-coluna ou látice; e a segunda, que não é incompatível com a primeira, é utilizar análises que incorporem diretamente o efeito da dependência espacial no modelo.

A aplicação da estatística espacial tem sido avaliada como alternativa à análise de dados em programas de melhoramento genético de várias culturas (Costa et al., 2005; Duarte \& Vencovsky, 2005; Resende et al., 2006; Candido et al., 2009). Na análise espacial, utiliza-se uma matriz não diagonal de variâncias e covariâncias residuais. Essa matriz é definida a partir de modelos geoestatísticos que representam funções de covariâncias que descrevem a dependência espacial dos erros em função da distância entre as parcelas. Para essa análise, é necessário coletar informação sobre a posição das parcelas na área experimental - as coordenadas $(\mathrm{x}, \mathrm{y})$ ao centro de cada parcela -, para que seja possível calcular a distância entre parcelas.

Feres (2009) identificou dependência espacial entre erros de fraca à moderada intensidade, em ensaios do programa de melhoramento genético do feijoeiro, da Universidade Federal de Viçosa (UFV), com grau de dependência entre 0,09 e 0,52. Contudo, como a dependência espacial entre erros não é considerada nas análises usuais em blocos e em látice, assume-se que eles sejam independentes. Dessa forma, de acordo com o autor, os modelos de análise espacial apresentaram melhor ajuste que a análise em látice. Além disso, nos ensaios com dependência espacial moderada, os autores relataram classificações diferentes das famílias entre a análise em látice e a análise espacial. Para a intensidade de seleção de $20 \%$ das famílias mais produtivas, a coincidência entre as duas metodologias de análise variou de 66 a $83 \%$. Resultados semelhantes foram relatados por Duarte \& Vencovsky (2005), quanto à classificação de genótipos de soja (Glycine $\max$ L.), em que se observou $46 \%$ de coincidência entre análise em blocos e análise espacial, para intensidade de seleção de $25 \%$. Portanto, é importante que se determine qual metodologia de análise é mais eficiente para classificação de famílias, em programas de melhoramento genético. Uma alternativa para identificar a metodologia mais eficiente é utilizar dados simulados, em que a média verdadeira de cada família é conhecida sem erro e diferentes cenários podem ser considerados.

O objetivo deste trabalho foi avaliar a eficiência da análise estatística espacial, em comparação a análises usuais em blocos ao acaso e em látice, na classificação de famílias de feijoeiro (Phaseolus vulgaris), sob diferentes cenários de dependência espacial e de precisão experimental.

\section{Material e Métodos}

Foram constituídos 12 cenários, com quatro classes de dependência espacial (nula, baixa, média e alta) e três classes de precisão experimental (moderada, alta e muito alta). O número de famílias, a média de produtividade (645 g por parcela quadrada) e a variância residual ( $7.744 \mathrm{~g}^{2}$ por parcela quadrada) foram definidos com base em ensaios anteriores, instalados entre 2006 e 2008, no programa de melhoramento genético do feijoeiro da UFV.

As quatro classes de dependência espacial entre erros (nula, baixa, média e alta) foram constituídas com os valores de $0,10,20$ e $40 \mathrm{~m}$, respectivamente, para o parâmetro alcance do modelo teórico exponencial. Este modelo foi utilizado para descrever a covariância entre erros em função da distância entre parcelas. Conforme Duarte \& Vencovsky (2005), o modelo exponencial foi o que apresentou melhor descrição da dependência espacial dos erros, em experimentos 
de avaliação genética de plantas. O parâmetro alcance indica a distância entre parcelas a partir da qual os erros são independentes. Assim, quanto maior o valor desse parâmetro, maior a dependência espacial entre erros.

O modelo exponencial utilizado na simulação foi: $\operatorname{Cov}\left(e_{i}, e_{j}\right)=\sigma^{2}\left(e^{-3 h / a}\right)$, em que $\operatorname{Cov}\left(e_{i}, e_{j}\right)$ é a covariância entre resíduos das parcelas i e j; $\sigma^{2}$ é a variância residual; a é o parâmetro alcance; e h é a distância (m) entre as parcelas i e j.

As distâncias entre parcelas foram calculadas a partir das coordenadas $(\mathrm{x}, \mathrm{y})$ relativas ao centro das parcelas de um ensaio real para avaliação de 100 famílias de feijoeiro, instalado em látice $10 \times 10$, com três repetições. Esse ensaio foi utilizado como referência no processo de simulação.

Para constituir as três classes de precisão experimental (moderada, alta e muito alta), foram definidos três valores de acurácia seletiva (AS), respectivamente: $0,60,0,80$ e 0,95 .

As variâncias devidas às famílias $\left(\sigma_{\mathrm{f}}^{2}\right)$ foram calculadas a partir dos valores predefinidos de AS e da variância residual $\left(\sigma^{2}=7.744\right)$, por meio das expressões descritas por Resende \& Duarte (2007), que envolvem a estatística F, para efeito de família, e o coeficiente de variação relativo $(\mathrm{CVr})$ :

$$
\mathrm{F}=\frac{1}{1-\mathrm{AS}^{2}} ; \mathrm{CVr}=\sqrt{\frac{\mathrm{F}-1}{\mathrm{r}}} ; \sigma_{\mathrm{f}}^{2}=\left(\mathrm{CVr} \times \sigma^{2}\right)^{2} .
$$

A partir da combinação de quatro classes de dependência espacial e três classes de AS, foram formados 12 cenários de dados simulados, e cada cenário foi simulado 1.000 vezes. A simulação dos dados foi feita com o programa R (R Core Team, 2014).

Os dados simulados foram analisados de acordo com os seguintes modelos estatísticos: modelo 1 (análise em blocos), $\mathrm{y}_{\mathrm{ij}}=\mu+\mathrm{b}_{\mathrm{j}}+\mathrm{g}_{\mathrm{i}}+\varepsilon_{\mathrm{ij}}$; modelo 2 (análise em látice), $\mathrm{y}_{\mathrm{ijk}}=\mu+\mathrm{r}_{\mathrm{k}}+\mathrm{b}_{\mathrm{j}(\mathrm{k})}+\mathrm{g}_{\mathrm{i}}+\varepsilon_{\mathrm{ijk}}$; e modelo 3 (análise espacial), $\mathrm{y}_{\mathrm{ij}}=\mu+\mathrm{b}_{\mathrm{j}}+\mathrm{g}_{\mathrm{i}}+\varepsilon_{\mathrm{ij}}$. Nestes modelos: $\mathrm{y}_{\mathrm{ij}}$ é o valor observado para a família $i$, no bloco $j ; \mu$ é a constante associada a todas as observações; $b_{j}$ é o efeito fixo do bloco j; $\mathrm{g}_{\mathrm{i}}$ é o efeito aleatório da família i; $\varepsilon_{\mathrm{ij}}, \varepsilon_{\mathrm{ijk}}$ e $\varepsilon_{\mathrm{ij}}$ são os erros aleatórios associados às observações, assumindo independência $\left(\varepsilon_{\mathrm{ij}}\right.$ e $\left.\varepsilon_{\mathrm{ijk}}\right)$ e dependência espacial $\left(\varepsilon_{\mathrm{ij}}\right)$ entre os erros; $\mathrm{y}_{\mathrm{ijk}}$ é o valor observado para a família i, no bloco j dentro da repetição $\mathrm{k}$; $r_{k}$ é o efeito fixo da repetição $k$; e $b_{j(k)}$ é o efeito fixo do bloco $\mathrm{j}$ dentro da repetição $\mathrm{k}$.

Para os modelos 1 e 2, assume-se que os erros aleatórios são espacialmente independentes, de forma que $\mathrm{R}=\mathrm{I} \sigma_{\mathrm{e}}^{2}$, em que $\mathrm{R}$ é a matriz de variâncias e covariâncias dos erros; I é a matriz identidade; e $\sigma_{\mathrm{e}}^{2}$, é a variância residual.

No modelo 3, considera-se a estrutura de dependência espacial entre os erros, em que $\mathrm{R}=\mathrm{F} \sigma_{\mathrm{e}}^{2}$ é uma matriz não diagonal e estruturada, na qual $\mathrm{F}$ é a matriz formada pelos elementos da função de distância entre parcelas $\mathrm{f}(\mathrm{h})=\mathrm{e}^{-3 \mathrm{~h} / \mathrm{a}}$, com uso do modelo exponencial no qual o alcance é estimado para representar a variabilidade espacial entre resíduos de parcelas separadas por uma distância h. Em cada uma das 1.000 análises realizadas, os parâmetros do modelo exponencial (alcance e variância residual), foram estimados a partir dos dados simulados, bem como a média de cada família.

Uma vez que o efeito das 100 famílias foi simulado, a média de cada família foi obtida sem erro. Desse modo, a classificação real das famílias foi utilizada para avaliar a eficiência das metodologias de análise testadas, por meio da correlação de Spearman entre médias verdadeiras e estimadas.

Para o ajuste do modelo exponencial, utilizou-se a metodologia de máxima verossimilhança restrita (REML), na estimação dos componentes de variância e das médias de famílias, por meio do procedimento Proc Mixed do programa SAS (SAS Institute, Cary, NC, EUA), de acordo com a metodologia descrita por Duarte et al. (2005).

\section{Resultados e Discussão}

Para os cenários com precisão experimental muito alta, as médias das correlações de Spearman entre médias estimadas e médias verdadeiras foram altas e com valores bem próximos, entre 93,33 e 99,34\%. Ainda para este nível de precisão, os coeficientes de variação $(\mathrm{CV})$ para a correlação de Spearman foram baixos, de 0,18 a 1,62\% (Tabela 1). Esses baixos valores de $\mathrm{CV}$ refletem pequena variação entre os resultados nas 1.000 simulações realizadas. Com o aumento da dependência espacial, a média aumentou, enquanto o CV para a correlação de Spearman apresentou ligeira diminuição. Embora a análise espacial tenha apresentado maiores médias e menores $\mathrm{CV}$, os resultados foram muito próximos dos obtidos com as análises em blocos e em látice. Dessa forma, para experimentos com precisão experimental muito alta, em que a variância decorrente do efeito de famílias é relativamente alta, a classificação das famílias 
com base nas médias estimadas foi bem próxima da classificação verdadeira, independentemente do tipo de análise e da classe de dependência espacial.

Cargnelutti Filho et al. (2009) avaliaram a precisão experimental de 72 e 216 ensaios em blocos ao acaso, para classificação de genótipos de feijão e soja, respectivamente. Destes, apenas $33 \%$ dos ensaios de feijão e $16 \%$ dos ensaios de soja, apresentaram precisão experimental muito alta, com AS superior a 0,90. Esses resultados, em conjunto com os obtidos no presente trabalho, revelam que a análise em blocos pode ser eficiente para classificar corretamente os genótipos em uma percentagem relativamente pequena dos ensaios, e que, nestes ensaios, a análise em látice e espacial não melhora a eficiência de classificação dos genótipos. Para outras culturas, segundo Cargnelutti Filho \& Storck (2009) e Cargnelutti Filho et al. (2012a, 2012b), os ensaios com precisão experimental muito alta tampouco apresentaram grande frequência, de 42, 43 e 52\% para ensaios de avaliação de genótipos de milho (Zea mays L.), arroz (Oryza sativa L.) irrigado e cana-de-açúcar (Saccharum officinarum L.), respectivamente.

Para os cenários de alta a moderada precisão experimental, constatou-se maior influência do tipo de análise sobre a classificação das famílias, sendo

Tabela 1. Médias e coeficientes de variação (entre parênteses) da correlação de Spearman (\%) entre médias estimadas nas diferentes análises e médias verdadeiras das famílias, nos 12 cenários simulados sob quatro classes de dependência espacial e três classes de precisão experimental.

\begin{tabular}{lccc}
\hline Dependência espacial & Blocos & Látice & Espacial \\
\hline \multirow{4}{c}{ Precisão experimental muito alta } \\
Nula & $94,03(1,44)$ & $93,33(1,62)$ & $94,00(1,45)$ \\
Baixa & $94,90(1,39)$ & $96,10(1,04)$ & $97,89(0,52)$ \\
Média & $95,97(1,27)$ & $97,40(0,71)$ & $98,83(0,29)$ \\
Alta & $97,22(0,99)$ & $98,42(0,47)$ & $99,34(0,18)$ \\
\hline \multicolumn{4}{c}{ Precisão experimental alta } \\
Nula & $78,08(5,39)$ & $76,15(6,19)$ & $77,99(5,46)$ \\
Baixa & $80,17(5,82)$ & $83,94(4,45)$ & $90,76(2,47)$ \\
Média & $84,20(4,82)$ & $89,25(2,86)$ & $94,91(1,27)$ \\
Alta & $88,54(3,87)$ & $93,26(2,03)$ & $97,21(0,73)$ \\
\hline \multicolumn{4}{c}{ Precisão experimental moderada } \\
Nula & $57,57(12,54)$ & $55,15(13,41)$ & $57,48(12,56)$ \\
Baixa & $60,79(11,94)$ & $66,03(10,07)$ & $77,73(5,93)$ \\
Média & $66,00(10,72)$ & $74,54(7,35)$ & $86,48(3,39)$ \\
Alta & $73,71(8,80)$ & $82,87(4,81)$ & $92,32(1,96)$ \\
\hline
\end{tabular}

que a análise espacial foi a mais eficiente. As médias das correlações de Spearman foram de 88,54, 93,26 e $97,21 \%$, respectivamente, para as análises em bloco, em látice e espacial, nos cenários com alta precisão experimental e alta dependência espacial (Tabela 1). Para os cenários com moderada precisão experimental e alta dependência espacial, a eficiência da análise espacial foi ainda maior, com médias das correlações de Spearman de 73,71, 82,87 e 92,32\%, respectivamente para as análises em blocos, em látice e espacial.

$\mathrm{Na}$ avaliação de genótipos de diferentes culturas, grande parte dos ensaios tem precisão experimental de alta a moderada. Os ensaios com este grau de precisão experimental representaram 64\% dos ensaios com feijão (Cargnelutti Filho et al., 2009), 74\% dos ensaios com soja (Cargnelutti Filho et al., 2009), 52\% dos ensaios com milho (Cargnelutti Filho \& Stork, 2009), $54 \%$ dos ensaios com arroz irrigado (Cargnelutti Filho et al., 2012b) e 47\% dos ensaios com cana-de-açúcar (Cargnelutti Filho et al., 2012a). Estes resultados destacam a superioridade da análise espacial em relação à em blocos, na classificação correta de genótipos, para a maior parte dos ensaios de competição de cultivares nas principais culturas. Além disso, em situações com limitações para a instalação de ensaios em látice, podese instalar os ensaios em blocos ao acaso e utilizar a análise espacial para classificar as famílias com maior eficiência, na presença ou não de dependência espacial.

A análise espacial também apresentou os menores valores de CV para a correlação de Spearman, nas diferentes repetições de um mesmo cenário simulado (Tabela 1). Com o aumento da dependência espacial e a redução da precisão experimental, observaram-se maiores diferenças entre os resultados das análises testadas. Para os cenários com alta dependência espacial e baixa precisão experimental, os CV foram de $8,80,4,81$ e $1,96 \%$, respectivamente para as análises em blocos, em látice e espacial.

Nos cenários com dependência espacial nula e precisão experimental moderada, os três tipos de análises apresentaram resultados muito próximos, com médias para correlação de Spearman de 55,15 a $57,57 \%$, e para CV de 12,56 a 13,42\% (Tabela 1). Esses resultados são indicativos de que, mesmo na ausência de dependência espacial, a análise espacial apresenta eficiência de classificação das famílias similar às das análises usuais. 
Caetano (2013), em trabalho com dados simulados, concluiu que a abordagem com uso de modelos autorregressivos para considerar a dependência espacial aumenta a precisão e a acurácia de experimentos instalados em delineamento em blocos ao acaso e em quadrados latinos. Resultados similares foram obtidos por Rossoni (2011), em que a dependência espacial foi considerada na análise com a utilização de modelos geoestatísticos, semelhantemente à análise espacial realizada no presente trabalho. Storck et al. (2011) avaliaram 26 ensaios de competição de cultivares de feijão instalados em blocos ao acaso e concluíram que, em média, a acurácia seletiva aumentou de 0,82 para 0,89 com uso do método de Papadakis, que é um dos métodos da análise espacial.

Apesar desses resultados positivos para análise espacial, segundo Maia et al. (2013), o uso de modelos autorregressivos para descrever a dependência espacial entre erros proporcionou apenas pequenos ganhos em relação à análise em blocos. Os autores avaliaram ensaios para seleção de clones de laranja 'Pêra', e consideraram a produção de frutos e o volume de copa das plantas. Candido et al. (2009) também verificaram que a análise espacial, com o método de Papadakis e médias móveis, não melhorou a precisão experimental para avaliação de genótipos de cana-de-açúcar. Entretanto, esses autores consideraram dados de apenas um ensaio de competição.

Conforme Henderson (1984), o parâmetro estatístico mais importante no contexto da avaliação genotípica é a acurácia seletiva. Este parâmetro refere-se à correlação entre o valor genotípico verdadeiro do tratamento genético e aquele estimado, ou predito, a partir das informações dos ensaios. Vários autores destacaram que a acurácia seletiva é um parâmetro adequado para classificação da precisão experimental de ensaios de competição de genótipos de milho, feijão, soja, arroz e cana-de-açúcar (Resende \& Duarte, 2007; Cargnelutti Filho et al., 2009, 2012a, 2012b).

De acordo com Resende \& Duarte (2007), valores de acurácia seletiva superiores a $80 \%$ devem ser buscados, e ensaios com acurácia seletiva inferior a $60 \%$ não devem ser aproveitados. Para atingir uma acurácia seletiva ideal, de $95 \%$ ou mais, os valores da estatística $\mathrm{F}$ para efeito de cultivares devem ser iguais ou superiores a 10,26; por conseguinte, este pode ser um valor de referência para ensaios de avaliação de cultivares.
Os resultados de acurácia seletiva estimada (ASE) em função da estatística $\mathrm{F}$ para efeito de família foram muito próximos (Tabela 2) aos obtidos para a correlação de Spearman entre médias estimadas e verdadeiras para as famílias (Tabela 1). Esta coerência dos valores de ASE com a correta classificação das famílias é muito importante para a avaliação genética nas diferentes culturas, pois ela indica que a ASE é uma medida eficiente da correlação entre valores genotípicos verdadeiros e estimados, e deve ser utilizada como medida de precisão experimental nos ensaios de avaliação genética de plantas. Resende \& Duarte (2007) confirmaram que a acurácia seletiva é uma medida que facilita e agrega as informações mais relevantes sobre a precisão experimental, na avaliação genética de plantas. Cargnelutti Filho et al. (2009, 2012a, 2012b) também apontaram que as estatísticas acurácia seletiva e $\mathrm{F}$ para cultivares têm relação direta entre si e são mais adequadas do que o coeficiente de variação experimental e a diferença mínima significativa, na avaliação da precisão experimental em ensaios para avaliação de genótipos de feijão, soja, arroz e cana-de-açúcar.

Tabela 2. Médias e coeficientes de variação (entre parênteses) para acurácia seletiva estimada $(\mathrm{ASE})^{(1)}$ nas diferentes análises nos 12 cenários simulados sob quatro classes de dependência espacial e três classes de precisão experimental.

\begin{tabular}{lccc}
\hline Dependência espacial & Blocos & Látice & Espacial \\
\hline Nula & \multicolumn{3}{c}{ Precisão experimental muito alta } \\
Baixa & $0,95(0,0099)$ & $0,94(0,0112)$ & $0,95(0,0097)$ \\
Média & $0,96(0,0104)$ & $0,97(0,0072)$ & $0,99(0,0028)$ \\
Alta & $0,97(0,0101)$ & $0,98(0,0050)$ & $0,99(0,0014)$ \\
\hline \multicolumn{4}{c}{ Precisão experimental alta } \\
Nula & $0,98(0,0081)$ & $0,99(0,0032)$ & $0,99(0,0007)$ \\
Baixa & $0,78(0,0533)$ & $0,78(0,0600)$ & $0,80(0,0521)$ \\
Média & $0,82(0,0517)$ & $0,86(0,0364)$ & $0,93(0,0150)$ \\
Alta & $0,85(0,0469)$ & $0,90(0,0263)$ & $0,96(0,0076)$ \\
\hline \multicolumn{4}{c}{ Precisão experimental moderada } \\
Nula & $0,89(0,0365)$ & $0,94(0,0161)$ & $0,98(0,0038)$ \\
Baixa & $0,56(0,1897)$ & $0,56(0,2243)$ & $0,58(0,1843)$ \\
Média & $0,61(0,1714)$ & $0,68(0,1214)$ & $0,82(0,0506)$ \\
Alta & $0,67(0,1255)$ & $0,74(0,0713)$ & $0,89(0,0235)$ \\
\hline
\end{tabular}

${ }^{(1)} \mathrm{ASE}=(1-1 / \mathrm{F})^{0,5}$, em que F é a estatística para o efeito de família na análise de variância (Anova). 
Para os cenários com precisão experimental muito alta, as médias de ASE, nas diferentes análises e classes de dependência espacial, foram altas e apresentaram valores bem próximos, de 0,94 a 0,99 , e os coeficientes de variação foram baixos, de 0,07 a 1,12\% (Tabela 2). Segundo Resende \& Duarte (2007), ensaios com maior acurácia seletiva, em geral, são os que apresentam menor variância residual e maior variância genotípica. A acurácia seletiva é dependente da proporção relativa entre os coeficientes de variação experimental e de variação genotípica, bem como do número de repetições.

Com a redução na precisão experimental, também verificaram-se grandes reduções nas médias de ASE. Esse resultado era esperado, uma vez que os cenários simulados com menor precisão experimental referemse àqueles com menor variância devido ao efeito de família.

Para os cenários de alta e moderada precisão experimental, observou-se que a análise espacial apresentou melhores resultados de ASE, em comparação às análises usuais. As médias de ASE foram de 0,89, 0,94 e 0,98, respectivamente, para as análises em blocos, em látice e espacial, nos cenários com alta precisão experimental e alta dependência espacial. Nos cenários com moderada precisão experimental e alta dependência espacial, a ASE média foi de $0,74,0,84$ e 0,94 , respectivamente, para as análises em blocos, em látice e espacial (Tabela 2). A precisão experimental estimada com a análise espacial foi superior à com a análise em blocos, em aproximadamente 10 pontos percentuais, no cenário com conjunto de dados simulados com alta precisão experimental, e em 20 pontos percentuais no cenário com precisão experimental moderada. Esse resultado é semelhante ao encontrado para a eficiência da análise espacial em classificar corretamente as famílias.

Nos cenários com dependência espacial nula, as médias de ASE foram próximas às dos valores simulados $(0,95,0,80$ e 0,60$)$, independentemente do tipo de análise (Tabela 2). Contudo, para cenários com dependência espacial baixa, média ou alta, o tipo de análise resultou em grande influência sobre as estimativas de ASE. Nesses três cenários, as médias para esse atributo aumentaram com o aumento do grau de dependência espacial. Porém, a análise espacial foi a que apresentou maior precisão experimental, quantificada pela ASE, em todos os cenários com dependência espacial. Além disso, mesmo nos cenários com menor precisão experimental, a análise espacial foi mais eficiente que as análises usuais em blocos e em látice.

\section{Conclusões}

1. Para ensaios com precisão experimental muito alta ou com ausência de dependência espacial, a eficiência da análise espacial para classificação de famílias do feijoeiro (Phaseolus vulgaris) é semelhante à das análises usuais em blocos ao acaso e em látice.

2. Para ensaios com alta e, principalmente, com moderada precisão experimental, a análise espacial é mais eficiente que as análises usuais para classificação de famílias, quando os erros apresentam dependência espacial.

\section{Agradecimentos}

Ao Conselho Nacional de Desenvolvimento Científico e Tecnológico (CNPq) e à Fundação de Amparo à Pesquisa de Minas Gerais (Fapemig), pelo apoio no desenvolvimento deste trabalho.

\section{Referências}

CAETANO, E. do R.R. Análise de variância utilizando modelos autorregressivos em experimentos com dependência espacial. 2013. 115p. Dissertação (Mestrado) - Universidade Federal de Lavras, Lavras.

CANDIDO, L.S.; PERECIN, D.; LANDELL, M.G. de A.; PAVAN, B.E. Análise de vizinhança na avaliação de genótipos de cana-de-açúcar. Pesquisa Agropecuária Brasileira, v.44, p.1304-1311, 2009. DOI: 10.1590/S0100-204X2009001000014.

CARGNELUTTI FILHO, A.; BRAGA JUNIOR, R.L. do C.; LÚCIO, A.D.C. Medidas de precisão experimental e número de repetições em ensaios de genótipos de cana-de-açúcar. Pesquisa Agropecuária Brasileira, v.47, p.1413-1421, 2012a. DOI: 10.1590/S0100-204X2012001000001.

CARGNELUTTI FILHO, A.; MARCHESAN, E.; SILVA, L.S. da; TOEBE, M. Medidas de precisão experimental e número de repetições em ensaios de genótipos de arroz irrigado. Pesquisa Agropecuária Brasileira, v.47, p.336-343, 2012b. DOI: 10.1590/ S0100-204X2012000300004.

CARGNELUTTI FILHO, A.; STORCK, L. Medidas do grau de precisão experimental em ensaios de competição de cultivares de milho. Pesquisa Agropecuária Brasileira, v.44, p.111-117, 2009. DOI: 10.1590/S0100-204X2009000200001.

CARGNELUTTI FILHO, A.; STORCK, L.; RIBEIRO, N.D. Medidas da precisão experimental em ensaios com genótipos 
de feijão e de soja. Pesquisa Agropecuária Brasileira, v.44, p.1225-1231, 2009. DOI: 10.1590/S0100-204X2009001000003.

COSTA, J.R.; BUENO FILHO, J.S. de S.; RAMALHO, M.A.P. Análise espacial e de vizinhança no melhoramento genético de plantas. Pesquisa Agropecuária Brasileira, v.40, p.1073-1079, 2005. DOI: 10.1590/S0100-204X2005001100004.

CRESSIE, N.; HARTFIELD, M.N. Conditionally specified Gaussian models for spatial statistical analysis of field trials. Journal of Agricultural, Biological, and Environmental Statistics, v.1, p.60-77, 1996. DOI: 10.2307/1400560.

DUARTE, J.B.; VENCOVSKY, R. Spatial statistical analysis and selection of genotypes in plant breeding. Pesquisa Agropecuária Brasileira, v.40, p.107-114, 2005. DOI: 10.1590/ S0100-204X2005000200002.

FERES, A.L.G. Análise estatística espacial na avaliação de produtividade no melhoramento genético do feijoeiro. 2009. 79p. Dissertação (Mestrado) - Universidade Federal de Viçosa, Viçosa.

HENDERSON, C.R. Applications of linear models in animal breeding. Guelph: University of Guelph, 1984. 462p.

MAIA, E.; SIQUEIRA, D.L. de; CARVALHO, S.A. de; PETERNELLI, L.A.; LATADO, R.R. Aplicação da análise espacial na avaliação de experimentos de seleção de clones de laranjeira Pêra. Ciência Rural, v.43, p.8-14, 2013. DOI: 10.1590/ S0103-84782012005000134.

R CORE TEAM. R: a language and environment for statistical computing. Version 3.1.1. Vienna: R Foundation for Statistical Computing, 2014. Available at: <http://www.r-project.org/index. html $>$. Accessed on: 10 jul. 2014.

RAMALHO, M.A.P.; FERREIRA, D.F.; OLIVEIRA, A.C. de. Experimentação em genética e melhoramento de plantas. 3.ed. Lavras: UFLA, 2012. 305p.

RESENDE, M.D.V. de; DUARTE, J.B. Precisão e controle de qualidade em experimentos de avaliação de cultivares. Pesquisa Agropecuária Tropical, v.37, p.182-194, 2007.

RESENDE, M.D.V. de; THOMPSON, R.; WELHAM, S. Multivariate spatial statistical analysis of longitudinal data in perennial crops. Revista de Matemática e Estatística, v.24, p.147-169, 2006.

ROSSONI, D.F. Análise de variância para experimentos com dependência espacial. 2011. 108p. Dissertação (Mestrado) Universidade Federal de Lavras, Lavras.

STORCK, L.; RIBEIRO, N.F.; CARGNELUTTI FILHO, A. Precisão experimental de ensaios de feijão analisada pelo método de Papadakis. Pesquisa Agropecuária Brasileira, v.46, p.798-804, 2011. DOI: 10.1590/S0100-204X2011000800003. 